\title{
Bangla translation, adaptation and piloting of Edinburgh Postnatal Depression Scale
}

\author{
Kaniz Gausia ${ }^{1,2}$, Jena D. Hamadani ${ }^{1}$, Md. Manirul Islam¹, Mohammed Ali $^{3}$, Sultana Algin ${ }^{4}$, \\ Mohammed Yunus ${ }^{1}$, Colleen Fisher ${ }^{2}$ and Jacques Oosthuizen ${ }^{2}$ \\ ${ }^{1}$ ICDDR'B: Centre for Health and Population Research, Mohakhali, Dhaka 1212, Bangladesh; ${ }^{2}$ Public Health, \\ Edith Cowan University, Perth, WA; ${ }^{3}$ Centre for International Health, Curtin University of \\ Technology, Perth, WA; ${ }^{4}$ National Institute of Mental Health Hospital (NIMH), \\ Dhaka 1207, Bangladesh.Email: kgausia@yahoo.com
}

\begin{abstract}
Standardized questionnaires for screening common health problems in the community often need to be translated for use in non-English speaking countries. There is a lack of literature documenting the process of translation of such questionnaire/scale that would enable their application in cross-cultural settings and standardization of the procedure. This paper reports the process of translation into Bangla of the widely used Edinburgh Postnatal Depression Scale (EPDS) for use in Bangladesh. Three methods: forward translation, committee translation, and back translation were used to ensure the equivalence of the translated version. Both the English and Bangla versions were piloted among 10 social science graduates who were proficient in both the languages. The concurrence of each respondent between the two versions showed a correlation coefficient of $0.98(\mathrm{p}<0.01)$. The BlandAltman test also showed a high degree of agreement. The piloted version was also tested with 15 women in the postnatal period and found to be suitable for women with lower educational attainment. The documentation of the translation process and the lessons learnt would be helpful in similar settings where screening questionnaires need to be adapted for local use.
\end{abstract}

\section{Introduction}

Standardized screening questionnaires are low cost appropriate tools that are often used to detect common health problems in the community. As these questionnaires are usually available in English, it is always necessary to translate to the native language when used in non-English speaking countries. However, there are difficulties in the process of proper translation, and the lack of a local language version can become a barrier in assessing and reporting health conditions. Translated versions are needed in detecting health problems that will also allow cross-countries and as well as crosscultural comparisons ${ }^{1}$.

To achieve a good quality translated version of questionnaire, use of multiple methods of translation such as forward translation, backward translation and committee translation are desirable whenever possible ${ }^{2}$. However, there are some inherent limitations of each of these methods of translation. Forward translation directly translate questionnaire from its source language (for example English) to the target language (for example Japanese); and the backward translation reverses the translation from target language to its source language. Both forward and back translation deal with literal meaning only; they cannot guarantee the general equivalence of the original questionnaire and its translation. On the other hand, committee translation requires more logistic support in terms of time, money and involving experts in the translation process. But the advantage of committee translation is that it takes some responsibilities of ensuring the equivalence of a questionnaire and thus has been put forward to achieve a high quality translated version of a questionnaire. In committee translation, a group of expert get involve in translating questionnaires from source language to target language, emphasizing more importance on thematic translation in local languages rather than literal translation of a questionnaire ${ }^{3}$. In thematic translation, alteration or modification of 
questionnaire is allowed which is called adaptation, to capture the linguistic and cultural values in the translation process ${ }^{4}$.

However, a recent review on translated questionnaires required for quantitative survey did not find any strict guidelines followed in translation process ${ }^{5}$. Guidelines have been discussed by Flaherty and colleagues to check for five equivalences (content, semantic, technical, criterion and conceptual equivalence) while translating questionnaires from one language to another ${ }^{6}$. The content, semantic and technical equivalences are mostly related with translation process and thus discussed here, whereas the remaining two equivalences which assess the reliability and validity of the translated questionnaire against standard diagnosis are discussed elsewhere ${ }^{7}$.

Content equivalence ensures that all questions asked have salience in the local context and culture. For example, it is not prudent to ask a question about sexual practice among young unmarried girls in communities where culture and law do not permit any sexual relationship before marriage. Semantic equivalence is stressed both in terms of the denotative (i.e. literal) meaning and connotative (i.e. emotional) meaning of the word while translating a question from one language to another ${ }^{8}$.

Technical equivalence ensures that the method of instrument application is appropriate in the second culture. For example, a self-reporting system (paper-pencil test) of data collection could work well in Western culture but the same method may not be appropriate in Asian or African cultures where the majority of people are less educated and thus unable to fully understand what is being asked. In these cultures, interview methods of data collection could be more suitable and practical. The current paper is based on translation and adaptation process of the English language Edinburgh Postnatal Depression Scale (EPDS) into Bangla.

The EPDS is a 10-item questionnaire which asks about the feelings of a postnatal woman in the 7 days preceding the administration of the questionnaire ${ }^{9}$. Each item consists of a statement with four possible answer choices with a scoring system. The psychometric properties of EPDS have been tested against and shown high agreement with standard diagnostic methods such as DSM-IV and ICD-10 classification for postnatal depression (PND) both in developed and developing countries $^{10}$.

Translation and use of the EPDS has been reported in more than 20 languages, but few are from developing countries ${ }^{11}$. The Punjabi version of EPDS was developed and tested among Punjabi women living in London ${ }^{12}$. In India, the Goa study used the Konkani version of the EPDS ${ }^{13}$. It was felt that a Bangla translation of EPDS would be very useful, as 207 million people mostly in Bangladesh and India speak Bangla, the $5^{\text {th }}$ most commonly spoken language in the world ${ }^{14}$.

This paper reports the process of the development of an appropriate Bangla version of the EPDS during a recently concluded study in Bangladesh. Flaherty's guidelines for translation mentioned above were adapted and followed. The challenges faced and lessons learned during this translation process were felt to be of potential significance and benefit in the light of their relevance and applicability to similar situations in other cultures and countries.

\section{Materials and Methods}

The project was approved by the ethics committee of Edith Cowan University, Western Australia and the ethics review committee of ICDDR,B, Bangladesh. Consent was obtained from all study participants. Three translation methods were employed: forward translation, committee translation and back translation ${ }^{15,16}$.

Forward translation: The principal investigator (KG), whose mother language was Bangla and whose educational qualifications were obtained in the English medium, translated the EPDS from English to Bangla. This preliminary Bangla version of the EPDS was then made available to a local expert committee for discussion.

Committee translation: A bilingual local expert committee was formed at the beginning of the project. The committee comprised of a psychologist, a psychiatrist, a pediatrician, three public health physicians, and two educated laypersons from the community. The committee sat over several meetings and worked extensively on the preliminary Bangla EPDS to develop an intermediate workable Bangla version of the EPDS and named as Bangla Postnatal Depression Scale (EPDS-B). Repeated searches were made from an English-to-Bangla dictionary for the appropriate wording whilst equal attention was given to retaining the connotative meaning of the word to ensure that the EPDS was easily understandable by all classes of people in Bangladesh.

Back translation: This intermediate Bangla version was back translated from the target language to its source language by an experienced translator who 
was unaware of the project and had no knowledge about the EPDS. The back-translated version was then reviewed by two native English speaking health professionals to check for congruence with the original English version of the EPDS (please see acknowledgement section).

Pilot testing: Pilot testing was carried out on three stages as follows: (a) self-administration of both EPDS and EPDS-B, (b) interview and selfadministration of the EPDS-B, and (c) interviews only with the EPDS-B. These three stages of piloting of EPDS-B were undertaken sequentially to make the scale comprehensible, culturally acceptable and usable for the women in Bangladesh. A convenience sampling strategy was adopted for each of the stages, as described in more detail below.

- Self-administration of the EPDS and the EPDS-B: Envelopes containing the EPDS-B and EPDS questionnaires were serially numbered from 1-10. Ten female participants who worked at the Social and Behavioral Sciences Unit (SBSU) in ICDDRB were chosen conveniently. The participants were all university graduates, fluent in both Bangla and English, and were unaware of the PND study. The EPDS was administered during the morning tea break while the EPDS-B was applied during the afternoon tea break on the same day. It was ensured that the volunteers did not discuss items with each other while they were completing the questionnaires.

- Interviews and self-administration of the EPDS-B: Mothers of infants aged less than one year were recruited, after giving informed consent, from the Dhaka hospital of the ICDDRB and from the immunization clinic. A total of 11 women were recruited. The EPDS-B was administered to participating women by an interviewer followed by self- administration of the EPDS-B on the same day. Before selfadministration of the EPDS-B it was ensured that the mothers read and understood the instructions at the beginning of the Bangla questionnaire.

- Interviews only with the EPDS-B: Another group of women were administered the EPDS$\mathrm{B}$ by using interview technique only. These women were mothers of infants admitted to the Dhaka hospital of the ICDDR,B. Two team members completed a total of four interviews. During the interview, one team member conducted the interview while the other observed the session and took notes. Notes were taken on how easily the interviewer was interacting with the respondents, whether and how many times any question had to be repeated and the reaction of the respondents after listening to the question. Other points checked included whether the respondent needed clarification on any question, and whether the interviewer used a different word from that on the printed version. In addition to administration of EPDS-B items the following questions were asked from all the respondents: Did she understand all the words? Did she know what was being asked? Did she have any questions about it? How could the question be made clearer? Did any of the questions make her feel uncomfortable? These questions were asked to explore whether the participants comprehended the items and content of the items of EPDS-B clearly, and does the concepts of items of EPDS-B and method of administration of scale are culturally acceptable.

\section{Results}

Eight out of 10 volunteers returned completed EPDS and EPDS-B questionnaires. The Pearson's correlation coefficient was used to determine the relationship between the EPDS and EPDS-B total scores. A highly significant correlation was found between EPDS and EPDS-B total scores (Correlation coefficient was 0.981 and significance at 0.01 level). Figure 1 shows the scatter plot of total EPDS and EPDS-B scores that were positively correlated. Bland and Altman argued that the correlation coefficient only provides an impression about the strengths of association between two variables but that it was unable to predict about the agreement between two variables ${ }^{17,18}$. Therefore, the Bland and Altman test was carried out to measure the agreement between the English and Bangla versions of the EPDS (Figure 2).

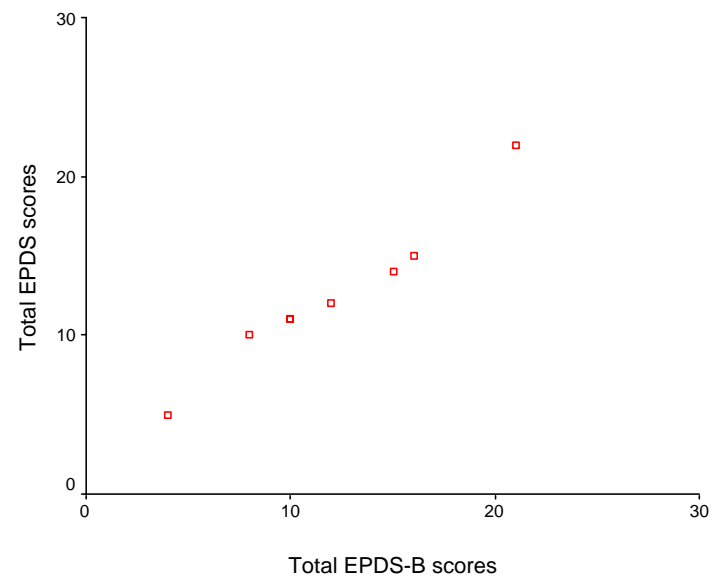

Figure 1: Scatter plot of EPDS and EPDS-B scores 


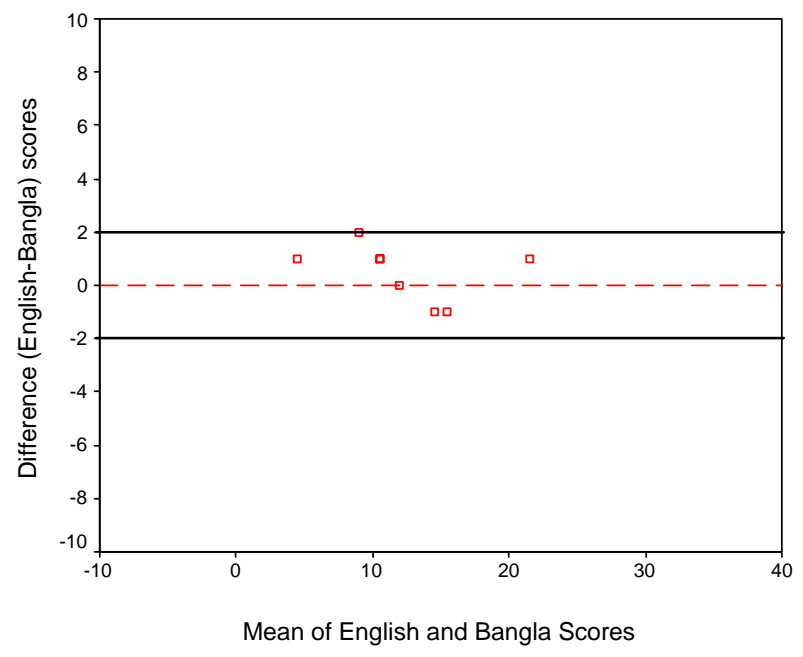

Figure 2: Bland-Altman plot of the difference between the English and Bangla EPDS against the mean English and Bangla scores in Bangladeshi women.

The Bland and Altman approach is based on a simple calculation technique together with graphical representation of the data. It helps in determination of the level of agreement between two sets of scores when comparing a new measurement technique, in this case the Bangla EPDS scale with an established one i.e. the English EPDS $^{9}$. The mean difference between the English and Bangla EPDS score was $0.50(\mathrm{SD}=1.07)$. The formula given for the limits of agreement was: mean difference $\pm 2 \mathrm{SD}$. The Bangla version of the EPDS could thus be 2.6 points above and -1.6 points below the English version. As can be seen in figure 2, of the 8 respondents, no score fell outside the limits of agreement, again supporting a high level of agreement between the two scores.

The women participating in this part of the pilot testing had a minimum of 5 years of school education and had infants ranging in age from 2 to 9 months. A significantly high correlation was observed between the total scores obtained by selfadministration and those obtained through interview using the EPDS-B scale. The Pearson's correlation coefficient was 0.752 at a 0.01 level of significance. Figure 3 shows a scatter plot of the total EPDS-B scores using the two different techniques of data collection. Finally, a Bland Altman calculation was also carried out to measure the level of agreement between the two methods (self-administration and interview). The mean difference between self-administration and interview EPDS-B scores was - 1.09 (SD = 4.11). Applying the Bland-Altman formula, the limits of interview scores in the EPDS-B scale may be 7.13 points above and -9.31 points below the selfadministered EPDS-B scores (Figure 4). The scores of all respondents, except for one, fell within the limits of agreement. These findings again support a greater level of agreement between selfadministration and interview scores.

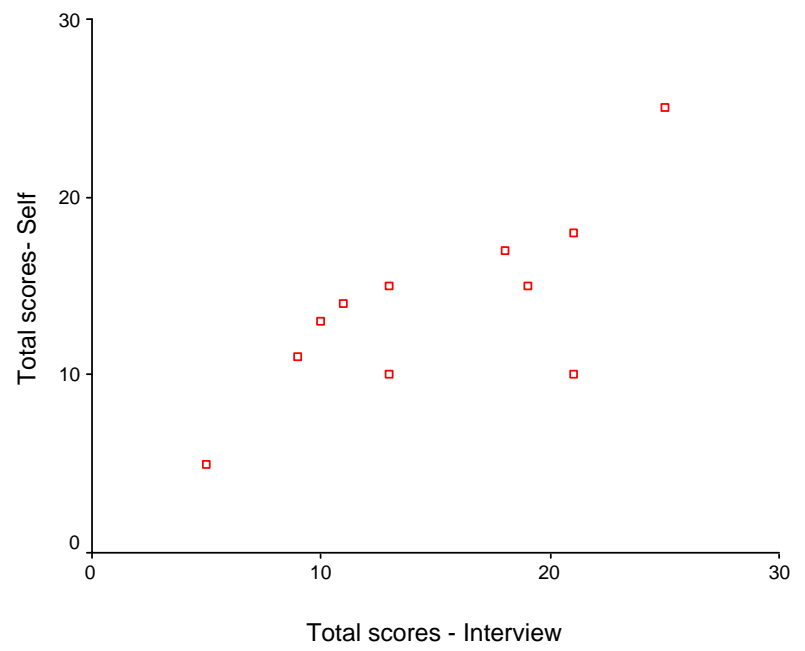

Figure 3: Scatter plot of EPDS-B self and interview scores

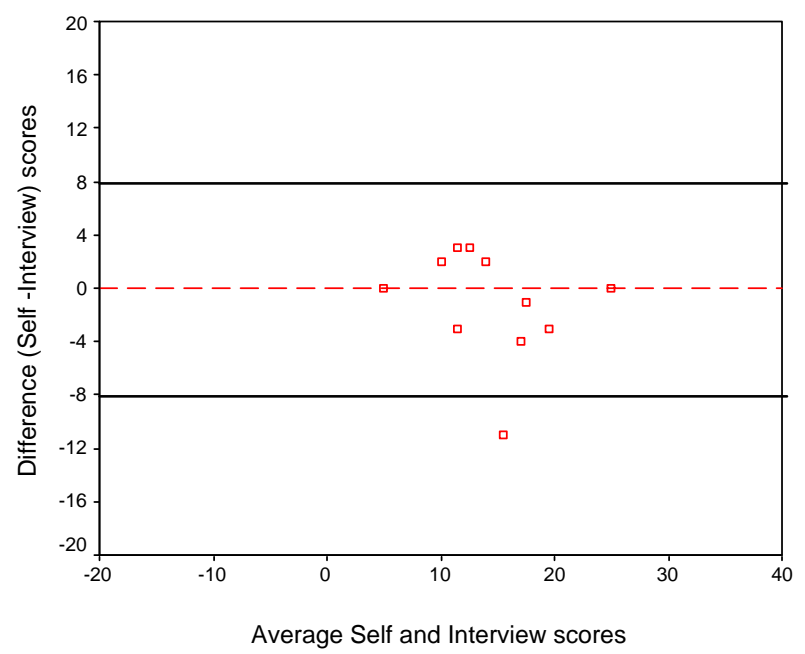

Figure 4: Bland- Altman calculation of EPDS-B scores in a sample of Bangladeshi women

Of the four interviews, the first two were quite difficult to carry out as respondents had to be addressed in the 'second person' (apni) during interviews to enable them to comprehend the questions, although the original questions were formulated in the 'first person' (ami). This was because of a cultural trait where questions asked by strangers often needed to be worded in the second person for the listener to comprehend and respond. This difficulty slowed the interview process and distracted both the interviewer and the respondent.

It was also noted in the first two interviews that the interviewer needed to read certain questions and their answer options several times before a respondent could understand them. The responses 
'less than before' and 'definitely less than before' appeared to confuse some of the respondents, as did 'yes, some times' and 'not very much'. In particular, question/item 2: "I have looked forward with enjoyment to things" and item 6: "Things have been getting on top of me" needed further explanation and clarification.

The questionnaires were modified to address the above problems. As a result, the remaining two interviews proceeded smoothly without any problems. Please see appendix for EPDS-B, the final Bangla version of the EPDS. From this exercise it became apparent that participants understood the questions when they were asked slowly and calmly in a private area. Additionally, numbering answer choice as for example, 1, 2, 3 and 4 is easier for study participants to respond to rather than repeating the content of these choices each time.

\section{Discussion}

In this paper we have presented the detailed translation procedure used in developing a Bangla version of the EPDS scale. The formation of a multidisciplinary research consultative group, translation, piloting and back translation proved to be very helpful in developing the Bangla version of the EPDS for screening PND in women in Bangladesh. The pilot study showed that it worked well, although some minor changes had to be made in finalizing the Bangla version to increase its technical equivalence.

Forward translation is an inexpensive and less time consuming method of translation compared to other methods of translation, for example, committee translation. In our study, the preliminary translation developed from forward translation helped in stimulating discussion among members of the local expert group in the committee translation stage. Not only did this process save time, it also provided an opportunity to assess and critique the preliminary translation of the EPDS. The local multi-disciplinary expert committee had long standing practical experience in translating questionnaires for use in the field situation and had a good reputation within the community. Both these factors impacted positively in maintaining the quality of the Bangla translation of the EPDS scale. Indeed, the quality of the translation depends heavily on qualifications, knowledge and cultural experience of the translators as well as their awareness about the research goal, concepts of interest and purpose of the items ${ }^{4,8}$.
In committee translation, more emphasis was given on thematic translation rather than word-for-word translation because word-for word translation can often be inadequate in addressing linguistic and cultural differences ${ }^{19}$. It was noted by the committee that item 2 and item 6 in the English EPDS scale were relatively difficult to express in Bangla language ${ }^{20}$. Through using sequential stages of piloting, namely the self-administration of the two language versions, both self-administration and interview administration of the Bangla version, and interview administration of the Bangla version alone, it was possible to refine and improve the translation procedure.

There was a high degree of concordance and agreement between the total scores obtained on both the versions in self-administration. Similarly, the results showed a high agreement of selfreported and interview methods of administration of EPDS-B, ensuring the technical equivalence of the Bangla version of EPDS. Thus, the selfreported EPDS scale can be applied by using interview technique in Bangladeshi culture.

It is hoped that the documentation of the rigorous scientific application of a rational translation process in developing the Bangla version of the EPDS will be useful in similar settings where screening questionnaires need to be translated and adapted for local use.

\section{Acknowledgements}

We would like to acknowledge the contribution of Dr. Anna Moore and Ms. Isabel for checking the English translation of the EPDS-B with the original EPDS, and Ms. Mahbuba for Back translation. Special thanks are also due to Ms. Sakila Yesmin for assisting in interviewing women and the EPI clinic chief- Dr. Jahan Afroze for allowing us to use the premises for interviews. This research was funded by the ICDDR,B: Centre for Health and Population Research and Department for International Development (DFID). The principal investigator of this study is supported by the Australian government International Postgraduate Research Scholarship (IPRS).

\section{References}

1. Hunt SM, Alonso J, Bucquet D, Niero M, Wiklund I, McKenna S. Cross-cultural adaptation of health measures. Health Policy 1991; 19: 33-44.

2. Capitulo KL, Cornelio MA, Lenz ER. Translating the short version of the Perinatal Grief Scale: Process and challenges. Appl Nurs Res. 2001; 14: 165-70.

3. Peters M, Passchier J. Translating instruments for cross-cultural studies in headache research. Headache 2006; 46: 82-91. 
4. Kristjansson EA, Desrochers A, Zumbo B. Translating and adapting measurement instruments for crosslinguistic and cross-cultural research: A guide for practitioners. Can J Nurs Res. 2003; 35: 127-42.

5. Bowden A, Fox-Rushby JA. A systematic and critical review of the process of translation and adaptation of generic health-related quality of life measures in Africa, Asia, Eastern Europe, the Middle East, South America. Soc Sci Med. 2003; 57: 1289-306.

6. Flaherty JA, Gaviria FM, Pathak D, Mitchell T, Wintrob R, Richman JA, Birz S. Developing instruments for cross-cultural psychiatric research. J Nerv Ment Dis. 1988; 176: 257-63.

7. Gausia K, Fisher C, Algin S, Oosthuizen J. Validation of the Bangla version of the Edinburgh Postnatal Depression Scale for Bangladeshi sample. J Reprod Infant Psychol. 2007; 25: 308-15.

8. Beck CT, Bernal H, Froman RD. Methods to document semantic equivalence of a translated scale. Res Nurs Health 2003; 26: 64-73.

9. Cox JL, Holden JM, Sagovsky R. Detection of postnatal depression. Development of the 10-item Edinburgh Postnatal Depression Scale. Br J Psychiatry 1987; 150: 782-86.

10. Eberhard-Gran M, Eskild A, Tambs K, Opjordsmoen S, Samuelsen SO. Review of validation studies of the Edinburgh Postnatal Depression Scale. Acta Psychiatr Scand. 2001; 104: 243-49.

11. Cox J, Holden J. Perinatal mental health: A guide to the Edinburgh Postnatal Depression Scale. London, The Royal College of Psychiatrist, 2003.
12. Werrett J, Clifford C. Validation of the Punjabi version of the Edinburgh postnatal depression scale (EPDS). Int J Nurs Stud. 2006; 43: 227-36.

13. Patel V, Rodrigues M, DeSouza N. Gender, poverty, and postnatal depression: A study of mothers in Goa, India. Am J Psychiatry 2002; 159: 43-47.

14. O'Neil D. 2006. Retrieved 23 July, 2007, from http://anthro.palomar.edu/language/language_1.htm

15. Maneesriwongul W, Dixon JK. Instrument translation process: A methods review. J Adv Nurs. 2004; 48: 175-86.

16. Jones PS, Lee JW, Phillips LR, Zhang XE, Jaceldo KB. An adaptation of Brislin's translation model for crosscultural research. Nurs Res. 2001; 50: 300-04.

17. Bland JM, Altman DG. Statistical methods for assessing agreement between two methods of clinical measurement. Lancet 1986; 1(8476): 307-10.

18. Bland JM, Altman DG. Measuring agreement in method comparison studies. Stat Methods Med Res. 1999; 8: 135-60.

19. Hilton A, Skrutkowski M. Translating instruments into other languages: Development and testing processes. Cancer Nurs. 2002; 25: 1-7.

20. Fuggle P, Glover L, Khan F, Haydon K. Screening for postnatal depression in Bengali women: Preliminary observations from using a translated version of the Edinburgh Postnatal Depression Scale (EPDS). J Reprod Infant Psychol. 2002; 20: 71-82. 